\title{
STABILITY OF THE NULL SOLUTION OF PARABOLIC FUNCTIONAL INEQUALITIES
}

BY

\author{
RAY REDHEFFER ${ }^{1}$ AND WOLFGANG WALTER
}

\begin{abstract}
Uniqueness and stability theorems are established for coupled systems of parabolic differential equations which may involve a Volterra-type dependence on the past history of the process. We allow retarded or deviating arguments, convolution-type memory terms, and strong coupling. (This means that all the space derivatives up to a given order can occur in all the equations.) Our results for strong coupling depend on the concept of "admissible monomial" which is here introduced for the first time and has no counterpart in the linear case. It is possible for uniqueness to fail in general, but to be restored (relative to a tolerably large class of functions of $(x, t))$ if a single solution independent of $x$ exists. Another curious feature of these theorems, depending again on the concept of admissible monomial, is that conditions for uniqueness can involve derivatives of order much higher than those occurring in the equation. Examples given elsewhere show that the results are, in various respects, sharp. Thus, the seemingly peculiar hypotheses do not arise from deficient technique, but from the actual behavior of strongly coupled systems. The paper concludes with a new method of dealing with unbounded regions for the difficult case in which the functional occurs in the boundary operator as well as in the differential equation.
\end{abstract}

0. Historical introduction. As the reader will recall, a powerful method of getting inequalities of the form $u(x, t) \leqslant \rho(x, t)$ for solutions $u$ of parabolic inequalities was introduced by Nagumo in 1939 and rediscovered by Westphal in 1949. The essence of the method is to choose $\rho$ so that $\rho(x, 0)>u(x, 0)$ and so that a contradiction is obtained at the first value $t$ such that $u(x, t)=\rho(x, t)$ for some $x$. Such a point $(x, t)$ is called a Nagumo point. The subject of this paper has its origin in certain extensions of this basic procedure.

(a) Inspired by early work of Max Müller (1926), Szarski and Mlak extended the method to weakly coupled systems in 1955, 1957 and 1959. Here one considers the first value $t$ such that the $k$ th component $u^{k}(x, t)=\rho(x, t)$ for some $x$ and some $k$. (b) It was observed by Redheffer in 1963 that the Nagumo procedure applies to equations containing functionals $u \rightarrow F u$, provided the functional is monotone and of Volterra type; that is, provided $(F u)(x, t)$ can be estimated from above by use of $\sup _{\xi} \sup _{\tau<t} u(\xi, \tau)$. (c) In 1975 Nickel introduced a novel approach to the theory of strongly coupled systems depending on the fact that, in a suitable function class, the space derivatives $D u$ can be estimated at $(x, t)$ by means of $\sup _{\xi}|u(\xi, t)|$. (The possibility of such estimation in one variable follows from inequalities of Landau, Bernstein and Kolmogorov.) Nickel's ingenious method [3]-[5] is, first, to substitute

Received by the editors March 6, 1979.

1980 Mathematics Subject Classification. Primary 35K55, 35R10, 35R45.

${ }^{1}$ Supported by NSF Grant No. MCS 77-03570.

(C) 1980 American Mathematical Society $0002-9947 / 80 / 0000-0510 / \$ 05.50$ 
the Bernstein or Kolmogorov estimate into the error formula which results from the Nagumo procedure, then to take the sup over $x$, and finally to get an explicit estimate from the resulting integral inequality. A generalization of this argument, based upon the classical theory of Volterra integral equations, is given in [9]. (d) In an effort to bring these results (c) into contact with the older methods (a), (b), the authors developed a comprehensive theory of two-sided inequalities. Here the goal is $|u(x, t)| \leqslant \rho(x, t)$ rather than the one-sided estimate $u^{k}(x, t)<\rho(x, t)$, and the appropriate measure of magnitude is

$$
|u|_{t}=\sup _{\xi}|u(\xi, t)| \quad \text { or } \quad|u|_{t, t}=\sup _{\xi} \sup _{\tau<t}|u(\xi, \tau)|
$$

as the case may be. The latter is used when $\rho(x, t)$ is increasing in $t$, the former when $\rho$ is unrestricted. These theorems are the first known to us which are directly applicable to strongly coupled systems containing Volterra functionals. Nevertheless, in the case of a bounded region, the method requires only minor changes from the procedure (a), (b); cf. proof of Theorems 1 and 3 for a bounded region, as outlined below. (e) What we have described so far is merely the formal basis for a theory of parabolic inequalities containing functionals, and does not come to grips with the main problems. The main problems are associated with the use of an unbounded region in the comparison argument and with the use of a general region in the Kolmogorov-type inequalities. The trouble with an unbounded region is that $\rho$ has to be unbounded, as a rule, to control $u$ at distant points; but then $|\rho|_{t}=\infty$, giving no estimate for $|u|_{t}$. In the Kolmogorov inequalities over general regions, trouble is encountered from the boundary.

These difficulties are essential. For the comparison inequality one has to impose certain growth restrictions on the coefficients, and without these restrictions, the purported extension to unbounded regions is false. For the Kolmogorov inequalities one has to impose an interior cone condition, and also to change the basic form of the inequality. Without such precautions, the extension to general regions is false.

These difficulties are overcome, at least to our own satisfaction, in the papers [9]-[12], to which this paper is a sequel. Since we wish to deal with what we perceive as the principal technical problems, all results here are formulated for general regions which need not be bounded.

1. Summary of results. With the introduction of the expression $|u|_{t}$ above, the theory of coupled systems appears in a new light. Namely, the undifferentiated component $u^{k}$ is displayed in the $k$ th equation along with $u_{x}^{k}$ and $u_{x x}^{k}$, and the other components $u^{j}$ are estimated at the Nagumo point by $|u|_{t}$ rather than by $u^{k}(x, t)$ as is customary. With no increase in complexity (and indeed, with the same comparison equation for $\rho$ ) the results apply to a much wider class of problems, including problems containing functionals such as $u \rightarrow D u$.

Another novelty of the present treatment is that a distinction is made between those aspects of the problem that depend on the past and those that do not. To this end, we introduce a memory function $\mu(t) \geqslant 0$ and a measure of magnitude 


$$
|u|_{\mu, t}=\sup _{\xi} \sup _{t-\mu(t)<\tau<t}|u(\xi, \tau)|
$$

in addition to the measure $|u|_{t}$ mentioned above. The latter corresponds to $\mu(t)=0$; that is, to memory 0 . Use of two memory functions is reminiscent of the two-timing approach of ten seen in the study of asymptotic behavior for parabolic equations, but it has a different motivation here. Theorem 1 , which gives a general comparison inequality in this setting, is supported by three corollaries asserting local, global and asymptotic stability. These would apply to a much smaller class of problems if we used only a single memory function $\mu$.

Our next result comes to grips with a basic difficulty in the theory of strongly coupled systems; namely, the comparison equation usually involves fractional powers of $\rho$ and does not even lead to uniqueness. (An exception arises in connection with the Bernstein class introduced into these problems by Nickel [3]-[5], for which an existence theory is developed in [8]. We believe, however, that this class is appropriate only for linear systems whose coefficients are independent of the Bernstein variable.)

The problem of fractional exponents is avoided here by the concept of admissible monomial. The gist of the matter is that, if the terms in the space derivatives $D u$ are of sufficiently high degree, then the exponents in the comparison equation are $>1$ and one gets not only uniqueness but local, global or asymptotic stability. These results, given in Theorem 2 and in the accompanying remarks, apply to problems of current interest, including the strongly coupled systems for fluid flow obtained in [2].

According to an often-quoted remark of Nirenberg, "most results for nonlinear problems are still obtained via linear ones, i.e. despite the fact that the problems are nonlinear and not because of it." By contrast, the results associated with admissible monomials make essential use of nonlinearity and do not have any counterparts in the linear case. These results cannot be applied, in general, to assess the difference of two solutions $u-v$, but they can be so applied if $v$ depends on $t$ alone. Thus we get some novel uniqueness theorems, to the effect that certain classes of strongly coupled systems cannot generate any spontaneous dependence on $x$ (despite the failure of uniqueness in general) if a single solution independent of $x$ exists. This discussion is prefaced by an extension of Theorem 1 to allow Neumann-type boundary conditions, $u_{\nu}=0$. Such conditions are natural for solutions of the form $v(t)$.

It turns out that the question whether a monomial is admissible (and hence, the question whether uniqueness holds) depends on the continuity class over which the monomial is considered. A monomial in the elements of $D u$ of order as low as 2 might be admissible relative to the class $C^{1000}$ but not relative to $C^{999}$. Examples given in [13] show that this reflects a genuine property of strongly coupled systems and that our conditions for a strongly coupled system not to generate any spontaneous dependence on $x$ are, basically, sharp.

The paper concludes with a new method of reducing estimation in an unbounded region to that in a bounded region. The main theorem is about certain 
classes of functions, not about differential equations, and it has applications other than the corollary presented here.

2. A summary of notation. In this paper $x \in R^{n}, t \in R$ and for simplicity $G=\Omega \times(0, \infty), G_{0}=\Omega_{0} \times\left[T_{0}, \infty\right), \Gamma=G_{0}-G$, where $\Omega$ is a domain in $R^{n}, \Omega_{0}$ is a closed subset of $R^{n}$ containing $\Omega$, and $T_{0} \leqslant 0$. We refer to $G$ as the parabolic interior and to $\Gamma$ as the parabolic boundary; when $\Omega_{0}=\bar{\Omega}$ and $T_{0}=0$ this terminology is standard. To distinguish conditions which hold for $t<T$ from those which hold for $t<\infty$ we define

$$
G(T)=\Omega \times(0, T], \quad G_{0}(T)=\Omega_{0} \times\left[T_{0}, T\right], \quad \Gamma(T)=G_{0}(T)-G(T) .
$$

The class $Z^{m}$ of admissible functions is the class of functions $u: G_{0} \rightarrow R^{m}$ which are bounded in $G_{0}(T)$ for each $T$, continuous in $G_{0}$, twice differentiable with respect to $x$ in $G$, and which have a left-derivative with respect to $t$ in $G$. Derivatives are denoted as usual by $u_{t}, u_{x}, u_{x x}$, the values being respectively in $R^{m}$, $\left(R^{n}\right)^{m},\left(S^{n}\right)^{m}$ where $S^{n}$ is the set of real symmetric $n$ by $n$ matrices. Monotonicity with respect to a matrix argument is denoted by an arrow; for instance, given $f$ : $R \times S^{n} \rightarrow R$ the notation $f\left(t, u_{x x} \uparrow\right)$ means

$$
f\left(t, u_{x x}+s\right) \geqslant f\left(t, u_{x x}\right) \geqslant f\left(t, u_{x x}-s\right), \quad(x, t) \in G, s \geqslant 0 .
$$

Here $u_{x x}=u_{x x}(x, t)$ and the inequality $s \geqslant 0$ is interpreted as usual by quadratic forms.

As norm for the vector $z \in R^{d}$ it is convenient to use

$$
|z|=\max \left(\left|z^{1}\right|,\left|z^{2}\right|, \ldots,\left|z^{d}\right|\right) \text {. }
$$

This applies to matrices as well as vectors; e.g., if $z \in S^{n}$ then $d=n^{2}$. With $|\cdot|$ as above and $u \in Z^{m}$ we define

$$
\begin{aligned}
|u|_{t} & =\sup \left\{|u(\xi, t)|:(\xi, t) \in G_{0}\right\}, \\
|u|_{\mu, t} & =\sup \left\{|u(\xi, \tau)|:(\xi, \tau) \in G_{0}, t-\mu(t)<\tau<t\right\}
\end{aligned}
$$

where $\mu$ is a given function $R_{+} \rightarrow R_{+}$which remains fixed throughout the discussion. It is supposed that $t-\mu(t) \geqslant T_{0}$ for $t \geqslant 0$, or that $T_{0}=-\infty$ if $t-\mu(t)$ is not bounded below. Note that $|u|_{t}=|u|_{0, t}$. This use of the subscript $t$ on the symbol $|\cdot|$ should be distinguished from its use to denote partial differentiation.

The statement of theorems is simplified by the following:

Notational convention. The letters $\delta, \varepsilon, \rho$ denote continuous functions $R \rightarrow R_{+}$ which are constant on $(-\infty, 0]$. The letter $N$ denotes an increasing function $R_{+} \rightarrow R_{+}$which can be large and can depend on the unknown function $u$.

3. An estimation theorem. Let an operator $P_{k}$ be defined for $u \in Z^{m}$ by

$$
P_{k} u=u_{t}^{k}-f_{k}\left(x, t, u^{k}, u_{x}^{k}, u_{x x}^{k} \uparrow, u(\cdot)\right), \quad k=1,2, \ldots, m,
$$

where $f_{k}$ is a function $G \times R \times R^{n} \times S^{n} \times Z^{m} \rightarrow R$. We assume that there is a function $\omega: R^{4} \rightarrow R$ such that

$$
\begin{aligned}
(\operatorname{sgn} z) f_{k}(x, t & , z, p, q, u(\cdot)) \\
& \leqslant \omega\left(t,|z|,|u|_{t},|u|_{\mu, t}\right)+N(t)|x||p|+N(t)|x|^{2}|q|
\end{aligned}
$$


for $k=1,2, \ldots, m,(x, t) \in G, p \in R^{n}, q \in S^{n}, u(\cdot) \in Z^{m}, z=u^{k}(x, t)$, where

$\omega(t, \alpha, \beta, \gamma \uparrow)$ is continuous, increasing in $\gamma$, and has

continuous partial derivatives with respect to $\alpha, \beta, \gamma$.

Inequality (1b) is needed in $G(T)$ for each $T$ subject to the side conditions

(i) $|x|>N(T),|p|<N(T)|x|^{-1},|q|<N(T)|x|^{-2}$,

(ii) $|x| \leqslant N(T), p=0, q=0$.

If only the coordinates $x_{j}$ for $j \leqslant l$ are unbounded in $\Omega$, and the rest are bounded, there is the further side condition

(iii) $p_{i}=q_{i j}=0$ for $i, j>l$.

Theorem 1. Let (1a)-(1c) hold, let $u \in Z^{m}$, and let $\rho_{t}>\omega\left(t, \rho, \rho, \rho_{\mu}\right)+\delta$ for $t>0, \rho \geqslant \varepsilon$ for $t \geqslant 0$, where $\rho_{\mu}(t)=\max \rho(\tau), t-\mu(t)<\tau<t$. Then $|P u|<\delta$ in $G$ and $|u| \leqslant \varepsilon$ in $\Gamma$ implies $|u| \leqslant \rho$ in $G_{0}$.

REMARK. For some applications it is important to know that the hypothesis $|P u| \leqslant \delta$ can be replaced by the weaker hypothesis $\left(\operatorname{sgn} u^{k}\right) P_{k} u<\delta, k=$ $1,2, \ldots, m$. This refinement results automatically from the proof. However, since (1b) is required only for $z=u^{k}(x, t)$, the refinement can also be obtained from Theorem 1 as it stands, by incorporating the error terms $\delta_{k}=P_{k} u,\left(\operatorname{sgn} u^{k}\right) \delta_{k}<\delta$, into $f$. A similar remark applies to results given below.

Proof (IN THE CASE of A bounded Region). If $\Omega$ is bounded, as supposed here, the proof of Theorem 1 is easy. By virtue of the conditions on $\omega$ we can assume that the two inequalities for $\rho$ are strict. If the conclusion does not hold there is a Nagumo point $(x, t) \in G$ and an index $k$ such that

$$
\left|u^{k}(x, t)\right|=\rho(t), \quad|u(\xi, \tau)| \leqslant \rho(\tau) \text { for } \tau<t .
$$

We assume $u^{k}(x, t)=+\rho(t)$; the discussion with - is similar. Then at $(x, t)$

$$
u_{t}^{k} \geqslant \rho_{t}, \quad u^{k}=\rho, \quad u_{x}^{k}=0, \quad u_{x x}^{k} \leqslant 0, \quad|u|_{t}=\rho, \quad|u|_{\mu, t}<\rho_{\mu} .
$$

Hence, at this point,

$$
P_{k} u \geqslant \rho_{t}-f_{k}(x, t, \rho, 0,0, u(\cdot)) \geqslant \rho_{t}-\omega\left(t, \rho, \rho, \rho_{\mu}\right)>\delta,
$$

which is a contradiction.

The above proof has been given to assist the reader in understanding Theorem 1, and also to make it plausible that the theorem is true. The result for an unbounded region is obtained by the iterative technique used in the proof of [12, Theorem 1]. Since only minor modifications are needed to adapt that argument to the case considered here, we do not repeat the details.

4. Stability. If $\rho$ is increasing then $\rho_{\mu}=\rho$ and Theorem 1 leads to an ordinary differential equation. By the regularity conditions on $\omega$ that equation is locally stable if $\omega(t, 0,0,0)=0$ and we get the following:

Corollary 1. Let the hypothesis of Theorem 1 hold with $\omega(t, 0,0,0)=0$. Then the null solution of the problem $P u=0, u(\Gamma)=0$ is locally stable relative to the class of functions $u \in Z^{m}$. That is, given any $\eta>0$ there exists $\lambda=\lambda(\eta ; T)>0$ such that $|P u|<\lambda$ in $G(T),|u|<\lambda$ in $\Gamma(T) \Rightarrow|u|<\eta$ in $G_{0}(T)$. 
It should be emphasized that $\lambda$ does not depend on $N$ or $\mu$, or on the a priori bound for $u$ in $G(T)$. Also $\lambda$ involves $P$ and $\Omega$ only insofar as $\lambda$ involves $\omega$.

If the conclusion holds in $G$ rather than just in $G(T)$ the problem is globally stable, and if $\lim |u(x, t)|=0$ under suitable hypotheses as $t \rightarrow \infty$, it is asymptotically stable. We shall discuss these matters in a special case which has an interesting bearing on the theory of strongly coupled systems. Let

$$
\omega(t, \alpha, \beta, \gamma)=-a(t) \alpha+b(t) \beta^{\sigma}+c(t) \gamma, \quad \sigma \geqslant 1, a>0, b \geqslant 0, c \geqslant 0,
$$

where $a, b, c$ are continuous functions $R \rightarrow R$ and $\sigma$ is constant. The differentialfunctional inequality for $\rho$ is then

$$
\rho_{t} \geqslant-a \rho+b \rho^{\sigma}+c \rho_{\mu}+\delta, \quad \rho \geqslant \varepsilon .
$$

In Corollaries 2 and 3 all relations involving lim, lim sup or lim inf pertain to the variable $t \rightarrow \infty$. The familiar $o$ and $O$ notation also expresses relationships as $t \rightarrow \infty$, under the understanding that these relationships hold uniformly in $x$ when applied to $u$ or $P u$. For example, $|P u|=o(a)$ means

$$
\lim _{t \rightarrow \infty} \frac{|P u|(x, t)}{a(t)}=0 \quad \text { uniformly in } x \in \Omega .
$$

Corollary 2 (Stability). Let $u \in Z^{m}$ and let $P$ satisfy the hypothesis of Theorem 1 with $\omega$ as in (2) and $\sigma>1$. Suppose further that $b=O(a)$ and $\lim \sup (c / a)<1$. Then for every $\eta>0$ there exists $\lambda=\lambda(\eta)>0$ such that $|P u|<\lambda a$ in $G,|u|<\lambda$ in $\Gamma \Rightarrow|u| \leqslant \eta$ in $G_{0}$.

COROllary 3 (ASYMPTOTIC STABILITY). Besides the hypotheses of Corollary 2, suppose one of the following conditions holds:

(i) $c=o(a)$ and $a$ is not integrable on $[0, \infty)$, or

(ii) $\lim$ inf $a>0$ and $\lim (t-\mu(t))=\infty$.

Then there exists $\lambda>0$ such that the implication $|P u|=o(a)$ in $G,|u|=o(1)$ in $\Gamma \Rightarrow|u|=o(1)$ in $G_{0}$ holds for every function $u \in Z^{m}$ satisfying $|P u|<\lambda a$ in $G$, $|u| \leqslant \lambda$ on $\Gamma$.

It will be seen that $\lambda$ depends on $a, b, c, \sigma$, in Corollary 3(ii) also on $\mu$, but not otherwise on $P, \Omega$, or $u$.

Proof. We write $\sigma=1+\kappa, \kappa>0$. To establish Corollary 2 , let $\lim \sup (c / a)=$ $1-3 \theta$ where $\theta>0$, and choose $\rho_{0}>0$ and $T>0$ so that $\rho_{0}<\eta, \rho_{0}^{\kappa} b(t)<\theta a(t)$, $c(t)<(1-2 \theta) a(t), t \geqslant T$. If $\delta<\lambda a$ with $\lambda=\theta \rho_{0}$ and if $\rho \equiv \rho_{0}$, the differential inequality (3) holds for $t>T$, since

$$
0>-a \rho_{0}+\theta a \rho_{0}+(1-2 \theta) a \rho_{0}+\theta \rho_{0} a .
$$

By Corollary 1 we can reduce $\lambda$, if necessary, so that $|u|<\rho_{0}$ holds in $G_{0}(T)$. Then the result follows by use of Theorem 1 in $G-G(T)$, with $\rho \equiv \rho_{0}$.

The proof of Corollary $3(\mathrm{i})$, is facilitated by the following elementary but useful lemma: 
LEMMA 1. Let $a, \phi$ be continuous functions $R_{+} \rightarrow R_{+}$, where $a$ is not integrable on $R_{+}$and $\phi$ is decreasing and $o(1)$. Then the solution of the initial-value problem

$$
\psi^{\prime}=(\phi-\psi) a, \quad \psi(0) \geqslant \phi(0)
$$

satisfies $\psi \geqslant \phi$ and $\psi=o(1)$.

We omit the trivial proof.

Let us now choose $\rho_{0}, 0<\rho_{0} \leqslant 1$, so that $2 \rho_{0}^{\kappa} b \leqslant a$. By Corollary 1 it is possible to choose $\lambda=\lambda(\omega)$ so that $|P u| \leqslant \lambda a,|u(\Gamma)| \leqslant \lambda \Rightarrow|u| \leqslant \rho_{0}$ and we suppose $\lambda$ so determined. Since $|P u|=o(a)$ and $c=o(a)$ there exists a positive function $\eta=$ $o$ (1) such that $c+\delta \leqslant \eta a$. The differential inequality (3) holds at any given value $t$ where

$$
\rho_{t} \geqslant-a \rho+\frac{1}{2} a \rho+\eta a, \quad \rho_{\mu}<\rho_{0} \leqslant 1 .
$$

Let the inequality $|u|=o(1)$ in $\Gamma$ be written in the form $|u|<\varepsilon, \varepsilon=o(1)$. Also let $\phi$ be a decreasing continuous function satisfying $\phi=o(1), \phi>2 \eta, \phi>\varepsilon$. Applying Lemma 1 with $a$ replaced by $a / 2$, we can find a function $\psi=o(1)$ such that $\psi_{t} \geqslant-\frac{1}{2} a \psi+\eta a, \psi \geqslant \varepsilon, \psi(0) \geqslant \rho_{0}$. Since $\psi=o(1)$ there is a last value, $T$, at which $\psi(T)=\rho_{0}$. We define $\rho=\rho_{0}$ for $t \leqslant T$ and $\rho=\psi$ for $t>T$. Since $|u| \leqslant \rho_{0}$ $=\rho(T)$, Theorem 1 applied in $G-G(T)$ gives $|u|<\rho=o(1)$.

We now establish Corollary 3(ii). Choose $\theta>0, A>0$ and $T$ so that $c<$ $(1-3 \theta) a$ and $a \geqslant A$ for $t \geqslant T$, and then choose $\rho_{0}$ so that $\rho_{0}^{k} b \leqslant a \theta, t>T$. The value $\lambda$ is now determined by Corollary 1 , as in the above proofs, so that $|u|<\rho_{0}$ in $G_{0}$.

The hypothesis $|P u|=o(a)$ in $G$ gives $\delta=\eta a$ where $\eta$ is continuous, positive, decreasing and $o(1)$. The hypothesis $|u|=o(1)$ in $\Gamma$ can be written $|u|<\varepsilon$ where $\varepsilon=o(1)$ and where, without loss of generality, the graph of $\varepsilon$ is convex; e.g., $\varepsilon^{\prime}<0$, $\varepsilon^{\prime \prime} \geqslant 0$. We shall choose for $\rho$ a piecewise linear function whose graph consists of line segments joining the successive points $\left(t_{n}, \rho_{n}\right)$. It should be thought that $t_{n}$ increases rapidly to $\infty$, and $\rho_{n}$ decreases to 0 . The precise values will be determined presently. However, among the conditions which we shall impose are

$$
t_{0} \geqslant T, \quad t_{n}<t_{n+1}, \quad t-\mu(t) \geqslant t_{n} \text { for } t>t_{n+1} .
$$

This latter is possible because $\lim (t-\mu(t))=\infty$.

The above conditions show that

$$
t_{n} \leqslant t \leqslant t_{n+1} \Rightarrow \eta(t) \leqslant \eta\left(t_{n}\right), \quad \rho_{\mu}(t) \leqslant \rho_{n-1}, \quad \rho(t)>\rho_{n+1},
$$

and also $\rho\left(t_{n}\right)>\varepsilon\left(t_{n}\right) \Rightarrow \rho(t)>\varepsilon(t)$. Hence, the differential inequality holds for the piecewise linear function $\rho$ on each of its segments if

$$
\frac{\rho_{n+1}-\rho_{n}}{t_{n+1}-t_{n}} \geqslant\left[-(1-\theta) \rho_{n+1}+(1-3 \theta) \rho_{n-1}+\eta\left(t_{n}\right)\right] a(t), \quad \rho_{n} \geqslant \varepsilon\left(t_{n}\right) .
$$

We now determine $\rho_{n}$ and $t_{n}$.

Choose $h, 0<h<1$, so that $(1-\theta) h^{2}-(1-3 \theta)-h \theta>0$. This is possible since the expression is positive when $h=1$. We define $\rho_{n}=\rho_{0} h^{n}, n=0,1,2, \ldots$, as well as $\rho(t)=\rho_{0}$ for $t<t_{0}$. Next we choose $s_{n}$ so large that

$$
\eta\left(s_{n}\right)<\theta \rho_{0} h^{n}, \quad \varepsilon\left(s_{n}\right)<\rho_{0} h^{n}
$$


and we require $t_{n} \geqslant s_{n}$ in addition to the conditions (4). Then (6) holds with $s_{n}$ replaced by $t_{n}$, and the bracket in (5) is positive. Because of this latter condition we can replace $a(t)$ in (5) by $A=\inf a$. A short calculation shows, then, that (5) holds if

$$
h-h^{2} \leqslant A\left(t_{n+1}-t_{n}\right)\left[(1-\theta) h^{2}-(1-3 \theta)-h \theta\right], \quad n>0 .
$$

(For $n=0$ the inequality has $(1-3 \theta) h$ on the right instead of $(1-3 \theta)$, hence is implied by the above.) If the sequence $\left\{t_{n}\right\}$ increases fast enough this holds, and the result follows from Theorem 1 in $G-G(T)$.

REMARK. Corollaries 2 and 3 require that $\varepsilon$ and $\delta$ be in some sense small, before any estimate is obtained. Such restrictions are not unexpected when the comparison equation is of degree $>1$, as it is in (3). For example, if $a$ and $b$ are positive constants and $\delta=c=0$, equation (3) has the constant solution $\rho_{0}=(a / b)^{1 / \kappa}$, where $\kappa=\sigma-1>0$. For $\rho(0)<\rho_{0}$ the solutions are $o(1)$, but for $\rho(0)>\rho_{0}$ the solutions tend to $\infty$ at a finite value, $T$, which depends on $\rho(0)$. If $\Omega=\mathbf{R}^{n}$ we could modify the assumptions on $u$ and $P$ in such a way that this $\rho$ would lead to an estimate for $u(x, t)$ from below. Hence, the instability represents a genuine aspect of the problem.

Of course when $a, b, c$ are variable, (3) may exhibit a great variety of behaviors. Some aspects of this behavior can be read off from the formula

$$
\frac{1}{\left[\rho(t) e^{A(t)}\right]^{\kappa}}=\frac{1}{\varepsilon^{\kappa}}-\int_{0}^{t} e^{-A(s)} b(s) d s, \quad A(t)=\int_{0}^{t} a(s) d s
$$

which gives the solution when $c=\delta=0, \rho(0)=\varepsilon, \sigma=1+\kappa$. It is helpful to distinguish two cases, according as $b e^{-A} \in L[0, \infty)$ or not. The formula then describes the function $\rho e^{A}$. To analyze the function $\rho$ we distinguish two further cases, according as $a$ is integrable on $[0, \infty)$ or not. Details of these developments are easy and are omitted. It should be mentioned, however, that the results apply to the strongly coupled system considered in Theorem 2 .

5. Admissible monomials. Partial derivatives with respect to $x$ (not $t$ ) are denoted by the usual indicial notation $D^{\alpha}$, and $D u$ is the vector of $m\left(1+n+\cdots+n^{d}\right)$ components which contains all $x$-derivatives $D^{\alpha} u,|\alpha|<d$. It is said that $\Omega \in$ $K(\theta, h)$ if each point $x \in \Omega$ is the vertex of a cone $C(x ; \theta, h) \subset \Omega$ which has altitude $h>0$ and vertex angle $2 \theta, 0<\theta<\pi / 2$. When $n=1, C(x ; \theta, h)$ is a line segment of length $h$.

The subclass of functions $u \in Z^{m}$ for which $D u$ is continuous in $x$ at each fixed $t>0$ is denoted by $C^{d} \cap Z^{m}$. For such functions we define $U_{k}(t)=$ $\sup \left\{\left|D^{\alpha} u(x, t)\right|:|\alpha|=k, x \in \Omega\right\}$ and state the following lemma [11]:

LEMmA 2. Let $\Omega \in K(\theta, h)$ and $u \in C^{d} \cap Z^{m}$. Then there exists a constant $J=J(d, n, \theta)$ (independent of $m, h, t)$ such that

$$
U_{k}(t) \leqslant J U_{0}(t)^{1-k / d} U_{d}^{*}(t)^{k / d}, \quad k=0,1, \ldots, d,
$$

where $U_{d}^{*}(t)=\max \left[U_{d}(t), h^{-d} U_{0}(t)\right]$. 
The hypothesis $U_{0}(t) \leqslant$ const for $0<t \leqslant T$ is already built into the definition of the class $Z^{m}$. If we introduce the further restriction $U_{d}(t)<$ const for $0<t<T$, or for $0<t<\infty$, we can use Lemma 2 to estimate functions of $D u$, much as the Bernstein and Landau-Kolmogorov inequalities are used in [3]-[6].

In general, Lemma 2 leads to an estimate with $U_{0}(t)^{p}$ where the exponent $p<1$, and the results do not even imply uniqueness, much less stability. The following definition, however, suggests a class of problems involving $\mathrm{Du}$ to which Theorem 1 applies:

Definition 1. Let $s_{j} \geqslant 0$ and $p_{j} \geqslant 0, j=0,1, \ldots, d$. The monomial

$$
M\left(s_{0}, s_{1}, \ldots, s_{d}\right)=s_{0}^{p_{0}} s_{1}^{p_{1}} \ldots s_{d}^{p_{d}}
$$

is said to be of type $(\sigma, \tau)$ if

$$
\sigma d=p_{0} d+p_{1}(d-1)+\cdots+p_{d-1}, \quad \tau d=p_{1}+2 p_{2}+\cdots+d p_{d} .
$$

The monomial is admissible if $\sigma \geqslant 1$ and strictly admissible if $\sigma>1$.

Note that $\sigma+\tau=p_{0}+p_{1}+\cdots+p_{d}$, which is the degree of $M$.

To get at the significance of this definition, suppose a monomial $F(D u)$ in the elements of $D u$ (i.e. a product of terms $D^{\alpha} u^{j}$ ) has coefficient 1 and $p_{k}$ factors of order $k, k=0,1, \ldots, d$; that is, $p_{k}$ factors $D^{\alpha} u^{j},|\alpha|=k$. With $s_{k}=U_{k}(t)$ it is obvious that $\sup _{x \in \Omega}|F(D u)|(x, t) \leqslant M\left(s_{0}, s_{1}, \ldots, s_{d}\right)$ where $M$ is the monomial considered in Definition 1. If $U_{0}(t) \leqslant h^{d} U_{d}(t)$, both expressions being finite, Lemma 2 gives

$$
|F(D u)| \leqslant J^{\sigma+\tau} U_{0}(t)^{\sigma} U_{d}(t)^{\tau}
$$

where $J=J(d, n, \theta)$. If $U_{0}(t)>h^{d} U_{d}(t)$ Lemma 2 gives

$$
|F(D u)| \leqslant J^{\sigma+\tau} U_{0}(t)^{\sigma+\tau}\left(h^{-d}\right)^{\tau} .
$$

In either case we get an estimate (const) $U_{0}(t)^{\sigma}$, where $\sigma>1$ if $M$ is admissible and $\sigma>1$ if $M$ is strictly admissible. The behavior is more favorable for small $U_{0}$ in the second case, but we shall generally have $U_{d}>>U_{0}$, so that only the first case is relevant.

In this example the $p_{j}$ are thought to be nonnegative integers. However, the choice $s_{j}=U_{j}(t) \geqslant 0$ allows any real $p_{j} \geqslant 0$ in $M$, whether integers or not, and Definition 1 has been formulated so as to take this into account.

6. Strongly coupled systems. Before stating a general theorem, we want to give some idea of the class of problems to which the foregoing theory applies. For functions $w \in Z^{1}$ let linear operators be defined by

$$
L_{k} w=a^{k}(x, t) w+\sum_{i=1}^{n} a_{i}^{k}(x, t) \frac{\partial w}{\partial x_{i}}-\sum_{i, j=1}^{n} a_{i j}^{k}(x, t) \frac{\partial^{2} w}{\partial x_{i} \partial x_{j}}
$$

where $\left(a_{i j}^{k}\right) \geqslant 0, k=1,2, \ldots, m$. We assume also that

$$
\left|a_{i}^{k}(x, t)\right| \leqslant N(t)|x|, \quad\left|a_{i j}^{k}(x, t)\right|<N(t)|x|^{2} \quad(|x|>N(t))
$$

for $(x, t) \in G$ and for all values of the indices $i, j, k$. A suitable class of operators $P_{k}$ is given by $P_{k} u=u_{t}^{k}+L_{k} u^{k}+F_{k}(x, t, D u)+G_{k}(x, t, u(\cdot))$ where the $G_{k}$ denote functionals. If $a^{k}(x, t) \geqslant a(t)>0$ and 


$$
\left|F_{k}(x, t, D u)\right| \leqslant b(t) U_{0}(t)^{\sigma}, \quad\left|G_{k}(x, t, u(\cdot))\right|<c(t)|u|_{\mu, t}
$$

then Theorem 1 applies with $\omega$ as in (2), and hence Corollaries 1-3 apply also.

To discuss (9), let the real vector $s=\left(s^{\alpha}\right)$ correspond to the elements of $D u=\left(D^{\alpha} u\right)$, and let $s_{k}=\max \left\{\left|s^{\alpha}\right|:|\alpha|=k\right\}, k=0,1, \ldots, d$, in correspondence with $U_{k}(t)$. Suppose $|F(x, t, s)| \leqslant B(t) \sum M_{i}\left(s_{0}, s_{1}, \ldots, s_{d}\right)$ (sum finite) where the $M_{i}$ are admissible and $B$ is continuous. If $u$ is restricted to the class of functions $u \in C^{d} \cap Z^{m}$ for which $U_{d}(t)<L$, where $L$ is constant, then the first inequality (9) holds with $b(t)=$ (const) $B(t)$ and with $\sigma>1$. If the $M_{i}$ are strongly admissible, $\sigma>1$.

Conditions on $G_{k}$ are readily established in specific cases. For example if

$$
G_{k}(x, t, u(\cdot))=\int_{\Omega} \int_{t-\mu(t)}^{t} \sum_{j=1}^{m} g_{k}^{j}(x, t ; \xi, \tau) u^{j}(\xi, \tau) d \tau d \xi
$$

where the $g_{k}^{j}$ are sufficiently smooth to ensure existence of the integral, the desired estimate (9) follows from

$$
\int_{\Omega} \int_{t-\mu(t)}^{t} \sum_{j=1}^{m}\left|g_{j}^{k}(x, t ; \xi, \tau)\right| d \tau d \xi<c(t) .
$$

Other examples are easily given.

We now generalize these remarks. Let $\boldsymbol{P}_{\boldsymbol{k}}$ be defined by

$$
P_{k} u=u_{t}^{k}-f_{k}\left(x, t, u^{k}, u_{x}^{k}, u_{x x}^{k} \uparrow, D u, u(\cdot)\right), \quad k=1,2, \ldots, m,
$$

where $f_{k}$ is a function $G \times R \times R^{n} \times S^{n} \times R^{m\left(1+n+\cdots+n^{d}\right)} \times Z^{m} \rightarrow R$. We assume there are positive continuous functions $a, b_{i}, c$ and finitely many admissible monomials $M_{i}$ such that

$$
\begin{aligned}
(\operatorname{sgn} z) f_{k}(x, t, z, p, q, s, u(\cdot)) \\
\leqslant-a(t) z+\sum b_{i}(t) M_{i}\left(s_{0}, s_{1}, \ldots, s_{d}\right)+c(t)|u|_{\mu, t} \\
\quad+N(t)|x|+N(t)|x|^{2}|q|
\end{aligned}
$$

for $k=1,2, \ldots, m,(x, t) \in G, p \in R^{n}, q \in S^{n}, s \in R^{m\left(1+n+\cdots+n^{d}\right)}, u(\cdot) \in Z^{m}$, $z=u^{k}(x, t)$. This is needed in $G(T)$ for each $T$ subject to side conditions (i)-(iii) of Theorem 1. As explained above, $s^{\alpha}$ corresponds to $D^{\alpha} u$ and $s_{j}=\max \left|s^{\alpha}\right|$ for $|\boldsymbol{\alpha}|=j$.

We shall establish the following:

THEOREM 2. Let $\Omega \in K(\theta, h)$, let (10a), (10b) hold, and let $L$ be a given continuous function $R \rightarrow R$ satisfying $L \geqslant 1$. Suppose $u$ is restricted to the subclass $(L)$ of functions $u \in C^{d} \cap Z^{m}$ satisfying $\sup \left\{\left|D^{\alpha} u(x, t)\right|: x \in \Omega,|\alpha|=d\right\}<L(t)$ for $(x, t) \in G$. Then

(i) If $u=0$ is a solution of the problem $P u=0$ in $G, u=0$ on $\Gamma$, then this solution is unique and locally stable relative to the class $(L)$.

(ii) If the monomials $M_{i}$ are strongly admissible and have types $\left(\sigma_{i}, \tau_{i}\right)$, and if the hypotheses of Corollaries 2 or 3 hold with

$$
b(t)=\sum b_{i}(t) L(t)^{\tau_{i}}, \quad \sigma=\min \sigma_{i},
$$

then the conclusion of Corollary 2 or 3 holds in the class $(L)$. 
Proof. If $U_{0}(t) \leqslant h^{d} L(t)$ either estimate (7) or (8) gives

$$
\left|M\left(s_{0}, s_{1}, \ldots, s_{d}\right)\right| \leqslant J^{\sigma+\tau} U_{0}(t)^{\sigma} L(t)^{\tau}
$$

where $s_{k}=U_{k}(t)$. Hence the expression $\Sigma b_{i} M_{i}$ in (10b) does not exceed $\sum b_{i}(t) J^{\sigma_{i}+\tau_{i}} J_{0}(t)^{\sigma_{i}} L(t)^{\tau_{i}}$ and the corresponding comparison equation for $\rho$ is $\rho>\varepsilon$ and

$$
\rho_{t} \geqslant-a(t) \rho+\sum b_{i}(t) J^{\sigma_{i}+\tau_{i}} L(t)^{\tau_{i}} \rho^{\sigma_{i}}+c(t) \rho_{\mu}+\delta .
$$

The condition $U_{0}(t) \leqslant h^{d} L(t)$ is assured if $\rho(t) \leqslant h^{d} L(t)$, which can be assumed for the $\rho$ used in Corollaries 2 and 3. We can also assume $\rho(t)<1$, so that the exponents $\sigma_{i}$ in (12) can be replaced by their minimum, $\sigma$. The constant $J$ does not affect the hypothesis $b=O(a)$, and Theorem 2 follows. For detailed estimates (12) should be used, but for the proof of stability (12) has no advantage over the simpler equation corresponding to $b(t)$ in Theorem 2 .

RemarKs. (a) The estimates underlying Theorem 2 remain valid even if the monomials $M_{i}$ are not admissible, and lead to results of the form $|P u|=o(1)$ in $G$, $|u|=o(t)$ on $\Gamma \Rightarrow|u|=o(t)$ in $G_{0}$ as $t \rightarrow 0+$. Aside from this one application, however, such estimates lose much of their impact unless $M_{i}$ are admissible. This is so because one could always put a bound on $|D u|$ independently of Lemma 2, and treat the problem as an inhomogeneous parabolic system.

Suppose, for example, that a strongly coupled system with $P$ as in (10a) contains first-order terms bounded by (const) $\left|D^{1} u\right|$ and is otherwise without complications, so that $a=c=0$. The following two comparison equations suggest themselves for the problem $P u=0$ in $G, u=0$ on $\Gamma$ :

(i) $\rho_{t} \geqslant(J L \rho)^{1 / 2}, \rho(0)=0$;

(ii) $\rho_{t} \geqslant J L, \rho(0)=0$,

where $J$ is constant. The first would be appropriate if $\left|D^{2} u\right|<L$ and $\left|D^{1} u\right|$ is assessed by Lemma 2 . The second would arise if $\left|D^{1} u\right|<L$ and the error produced by $D^{1} u$ is incorporated into the inhomogeneous term, $\delta$. Except for the behavior near $0+$, it would seem that the second (altogether trivial) result is preferable to the first. That is why the concept of admissible monomial has been emphasized here, and why only the behavior as $t \rightarrow 0+$ was emphasized in [12, Theorem 2].

(b) Our theorems on strongly coupled systems generally have a hypothesis to the effect that $\left|D^{\alpha} u\right|$ for $|\alpha|=d$ is not large, and a conclusion to the effect that $|u|$ is small. In that case Lemma 2 implies that the derivatives $\left|D^{\alpha} u\right|$ for $|\alpha|<d$ are also small. In particular, as $t \rightarrow \infty,\left|D^{\alpha} u\right|=O(1)$ for $|\alpha|=d,|u|=o(1) \Rightarrow\left|D^{\alpha} u\right|=o(1)$ for $|\alpha|<d$. Under mild additional conditions [11] the same holds for $|\alpha|=d$, so that finally $|D u|=o(1)$. This remark applies also to results given below.

(c) The main feature of Theorem 2 is not that it allows terms with retarded or deviating arguments, but that it allows strong coupling. The essential hypothesis for this latter application is that the monomials $M_{i}$ be admissible relative to the class $C^{d}$, where $d$ may have to be much larger than the order of the differential equation. Examples given in [13] show that, surprising as it may seem, this hypothesis is sharp. 
7. Mixed boundary conditions. If the normal derivative $u_{v}$ rather than $u$ is prescribed on the lateral part of the boundary, one must use a comparison function $\rho(x, t)$ depending on $x$. It might be thought that $D \rho$ would have to be well defined if $D u$ occurs in $P u$, but this is not the case. It suffices to have $\rho \in C^{2}$ even if $D$ is of arbitrarily high order, and $\rho$ need not be in the domain of $P$.

With the usual notation [15] for the interior normal derivative, including the convention that the interior normal $\nu$ lies in a hyperplane $t=t_{0}>0$, we introduce the following definition [7]:

DEFINITION 2. The boundary $\partial \Omega$ is regular if there exists a function $c(x) \in Z^{1}$ such that $c_{\nu}(x) \geqslant 1$ for $x \in \partial \Omega$ and

$$
0 \leqslant c(x) \leqslant C_{0}, \quad\left|c_{x}\right| \leqslant C_{1}, \quad\left|c_{x x}\right| \leqslant C_{2}
$$

for $x \in \Omega$. The constants $C_{j}$ are called the region constants relative to $c$.

Let

$$
P_{k} u=u_{t}^{k}-f_{k}\left(x, t, u^{k}, u_{x}^{k}, u_{x x}^{k} \uparrow, u(\cdot)\right)
$$

as in (1a), but assume instead of (1b)

$$
(\operatorname{sgn} z) f_{k}(x, t, z, p, q, u(\cdot))<\omega\left(t,|z|,|u|_{t},|u|_{\mu, t} ;|p|,|q|\right)
$$

where $\omega$ is continuous, is continuously differentiable with respect to the last five arguments, and is increasing with respect to the last four. As boundary conditions we assume

$$
u^{k} \operatorname{sgn} u^{k} \leqslant \varepsilon_{0} \quad \text { or } \quad-u_{\nu}^{k} \operatorname{sgn} u^{k}<\varepsilon, \quad(x, t) \in \Gamma,
$$

for $k=1,2, \ldots, m$ where $\varepsilon_{0} \geqslant 0$ and $\varepsilon \geqslant 0$ are constant. It is understood that one or the other condition holds for each $(x, t, k)$, but the question of which condition holds can depend on $(x, t, k)$. Since the normal derivative does not exist when $t \leqslant 0$, equation (13c) implies $|u(x, t)| \leqslant \varepsilon_{0}$ for $t \leqslant 0$, and this fact will be needed in the proof.

THEOREM 3. Let $\partial \Omega$ be regular and admit the region constants $C_{j}$, and let (13a)-(13c) hold with $u \in Z^{m}$. Suppose $\rho$ satisfies

$$
\rho_{t} \geqslant \sup _{0<\theta<\varepsilon C_{0}} \omega\left(t, \rho-\theta, \rho, \rho_{\mu} ; \varepsilon C_{1}, \varepsilon C_{2}\right)+\delta(t), \quad t>0,
$$

as well as $\rho(t) \geqslant \varepsilon_{0}+\varepsilon C_{0}$ for $t \geqslant 0$. Then $|P u|<\delta$ in $G$ implies $|u|<\rho$ in $G_{0}$.

In general, the right-hand side of the equation for $\rho_{t}$ has a favorable behavior as $\varepsilon \rightarrow 0$, and Theorem 3 can be used to establish uniqueness and local stability much as in Corollary 1. Since $\omega$ need not be increasing with respect to its second argument, the theorem also gives global stability in certain cases. The following is an example:

COROLlary 4. Let the hypothesis of Theorem 3 hold with

$$
\omega\left(t, \alpha, \beta, \gamma ; s_{1}, s_{2}\right)=-A \alpha+B \beta^{\sigma}+C \gamma+J_{1} s_{1}+J_{2} s_{2}
$$

where $A, B, C, J_{1}, J_{2}$ and $\sigma$ are positive constants and $A>C, \sigma>1$. Then given any $\eta>0$ there exists $\lambda>0$ such that the three conditions $\varepsilon_{0}<\lambda, \varepsilon \leqslant \lambda,|P u|<\lambda$ in $G$ together imply $|u| \leqslant \eta$ in $G_{0}$. 
In other words, the null solution of the problem $P u=0$ in $G, u^{k} u_{v}^{k} \geqslant 0$ in $\Gamma$ is globally stable, if we interpret $u^{k} u_{\nu}^{k} \geqslant 0$ to mean $u^{k}=0$ at points of $\Gamma$ where $u_{\nu}^{k}$ does not exist.

ProOF (FOR A BOUNDED REGION). We shall establish the stronger inequality $|u| \leqslant \rho-\varepsilon c$, where $c$ is the function in Definition 2. By the regularity condition on $\omega$, it can be assumed that the two inequalities for $\rho$ are strict. If $\Omega$ is bounded, and the desired conclusion $|u| \leqslant \rho-\varepsilon c$ does not hold, we can find a Nagumo point $(x, t)$ where, without loss of generality, $u^{k}(x, t)=\rho(t)-\varepsilon c(x),|u|_{\mu, t} \leqslant$ $|\rho(t)-\varepsilon c(x)|_{\mu, t} \leqslant|\rho|_{\mu, t},|u|_{t} \leqslant \rho(t)$. The boundary conditions show $(x, t)$ cannot be in $\Gamma$, and hence at $(x, t)$

$$
u_{t}^{k} \geqslant \rho_{t}, \quad u_{x}^{k}=-\varepsilon c_{x}, \quad u_{x x}^{k} \leqslant-\varepsilon c_{x x}, \quad(x, t) \in G,
$$

besides the conditions above. This gives a contradiction,

$$
P_{k} u \geqslant \rho_{t}-\omega\left(t, \rho-\varepsilon c, \rho, \rho_{\mu} ; \varepsilon C_{1}, \varepsilon C_{2}\right)>\delta .
$$

It should be observed that (13b) was used only for $|p|<\varepsilon C_{1},|q|<\varepsilon C_{2}$, a fact which greatly increases the scope of Theorem 3 . When the region is unbounded the function $c(x)$ is replaced by

$$
\tilde{c}(x)=(1+\alpha) c(x)+\alpha\left(1+x_{1}^{2}+\cdots+x_{n}^{2}\right)^{1 / 2}
$$

where $\alpha>0$ is arbitrarily small, and (13b) is needed for $|p|<\beta \varepsilon C_{1},|q|<\beta \varepsilon C_{2}$ with $\beta$ arbitrarily close to 1 . The proof is the same as in the case of a bounded region when the functional does not have any $x$ deviation, but requires the iterative technique of [12] in general.

Proof of Corollary 4. It suffices to note that the equations for $\rho$ and $\rho_{t}$ in Theorem 3 have the constant solution $\rho=\rho_{0}$, where $\kappa=\sigma-1$ and

$$
\rho_{0}\left(A-B \rho_{0}^{\kappa}-C\right) \geqslant \varepsilon\left(A C_{0}+J_{1} C_{1}+J_{2} C_{2}\right)+\delta, \quad \rho_{0}>\varepsilon_{0}+\varepsilon C_{0} .
$$

This not only gives Corollary 4 , but also shows how $\lambda$ depends on $(\Omega, \omega, \eta)$.

Corollary 4 applies to operators of the type considered in Theorem 2, where $B$ and $\sigma$ depend on the admissible monomials used there. Thus we get global stability for the null solutions of strongly coupled systems with mixed boundary conditions, including the usually troublesome condition $u_{\nu}=0$. The latter is of interest because it allows solutions depending on $t$ alone, as discussed next.

8. Problems with spatial homogeneity. If two equations involving an admissible monomial $\boldsymbol{M}$ are written for $u$ and for $\tilde{u}$, and subtracted, the resulting equation almost always involves monomials in $w=u-\tilde{u}$ which are not admissible. That is the reason why we have compared $u$ with the null solution, rather than with an arbitrary solution $\tilde{u}$. There is just one case in which the concept of admissible monomial can be used to estimate $u-\tilde{u}$. This is the case in which $\tilde{u}$ is a function of $t$ alone.

Let $M\left(s_{0}, s_{1}, \ldots, s_{d}\right)$ be a monomial of the type considered in Definition 1 , and suppose the exponent $p_{0}$ for $s_{0}$ is $p_{0}=0$ while at least one exponent $p_{j}>0, j>1$. Substituting appropriate derivatives $D^{\alpha} u^{j},|\alpha|=k$, for $s_{k}$, we get an expression which we denote by $M(u)$. Then $M(u \pm \tilde{u})=M(u)=M(u) \pm M(\tilde{u})$ whenever $\tilde{u}$ 
depends on $t$ alone. This is the basis for the behavior described above.

Consideration of $u(x, t)-\tilde{u}(t)$ gives a means of discussing the following question: Suppose a problem $P u=0$ with appropriate boundary conditions is spatially homogeneous, in the sense that it admits a solution $\tilde{u}(t)$ which is independent of $x$. Is every solution $u(x, t)$, in some reasonably large class, necessarily independent of $x$ ? In the case of strongly coupled systems, the question is whether the strong coupling can generate any dependence on $x$, when it is known a priori that a solution independent of $x$ exists. Instead of formulating a general theorem (as is easily done) we shall illustrate the main features in an example.

EXAmple. Let $m=2, n=1$ and write $(u, v)$ instead of $\left(u^{1}, u^{2}\right)$. For definiteness we take $G$ to be the first quadrant, $G=(0, \infty) \times(0, \infty)$, and $G_{0}=\bar{G}$. Let us consider the problem

$$
\left\{\begin{array}{l}
u_{t}=-u+a_{1}(x, t) u_{x x}+b_{1}(x, t) u v_{x x}^{2}+\int_{0}^{t} c_{1}(\tau) v(x+1, \tau) d \tau+g(t), \\
v_{t}=-v+a_{2}(x, t) v_{x x}+b_{2}(x, t)\left(u_{x} v_{x x}\right)^{2}+\int_{0}^{t} c_{2}(\tau) u\left(x^{2}, \tau\right) d \tau+h(t),
\end{array}\right.
$$

where the coefficients $a_{i}, b_{i}$ are bounded in $G(T)$ for each $T, a_{i}>0$, and $c_{i}$ are continuous. It is convenient to assume the following boundary conditions:

$$
u(x, 0)=u_{0}, \quad v(x, 0)=v_{0}, \quad \frac{\partial u}{\partial x}(0, t)=0, \quad \frac{\partial v}{\partial x}(0, t)=0 \quad(t>0) .
$$

If $g$ and $h$ are continuous, as now assumed, the problem has a solution $(y, z)$ depending on $t$ alone. When $g$ and $h$ are differentiable $(y, z)$ are given by the linear system

$$
\begin{aligned}
& y^{\prime \prime}+y^{\prime}=c_{1} z+g^{\prime}, \quad y(0)=u_{0}, \quad y^{\prime}(0)=g(0)-u_{0}, \\
& z^{\prime \prime}+z^{\prime}=c_{2} y+h^{\prime}, \quad z(0)=v_{0}, \quad z^{\prime}(0)=h(0)-v_{0} .
\end{aligned}
$$

In any case, we regard $(y, z)$ as known.

Upon substituting $u(x, t)=U(x, t)+y(t), v(x, t)=V(x, t)+z(t)$ into the original equations, one finds that $U, V$ satisfy the corresponding homogeneous system $\left(g=h=0\right.$ ) with the single exception that the expected term $b_{1} U V_{x x}^{2}$ in the first equation appears as $b_{1} u V_{x x}^{2}$. This is so because the factor $u$ must be incorporated into the coefficient and not into the monomial. The boundary conditions for $(U, V)$ are homogeneous.

The monomial $V_{x x}^{2}$ is admissible over the class $C^{d}$ if $d=4$, and strongly admissible if $d=5$. The monomial $\left(U_{x} V_{x x}\right)^{2}$ is strongly admissible in either case. Hence, the spatially homogeneous solution $(y, z)$ is unique over the class of functions $u \in C^{4} \cap Z^{m}$ for which $\left|D^{\alpha} u\right|$ is bounded in each $G(T),|\alpha|=4$. Furthermore there is local stability relative to the class $\left(L, L_{0}\right)$ for which $\left|D^{\alpha} u\right|<$ $L,|\alpha|=4$, and $|u| \leqslant L_{0}$. (The latter condition is needed to control the coefficient $b_{1} u$.) If

$$
\int_{0}^{\infty}\left|c_{1}(\tau)\right| d \tau<1, \quad \int_{0}^{\infty}\left|c_{2}(\tau)\right| d \tau<1
$$

and the coefficients $a_{i}, b_{i}$ are bounded in $G$, there is global stability relative to the class $\left(L, L_{0}\right)$ satisfying the above inequalities in $G$ with $d=5,|\alpha|=5$. 
Hence, the strong coupling does not generate any dependence on $x$ in a situation such as this. Furthermore, if $g, h$ and the boundary conditions are replaced by functions which show only a slight dependence on $x$, then the solution $u$ (restricted to an appropriate continuity class) will also show only slight dependence on $x$. Our main hypothesis $\left|D^{\alpha} u\right| \leqslant$ const for $|\alpha|=4$ or 5 is not unreasonable in this connection, since $D^{\alpha} y=D^{\alpha} z=0$ for all $|\alpha|>1$.

In conclusion, we mention that comparison with $(y, z)$ really is different from comparison with $(0,0)$. Local or global stability of the null solution requires $u \in C^{d} \cap Z^{m}$ with $d=2$ or 3 , respectively, rather than 4 or 5 , and no bound $|u| \leqslant L_{0}$ is needed. The fact that different aspects of this problem lead to four different continuity classes $C^{d}, d=2,3,4,5$, is connected with the phenomenon noted in §6; cf. also [13].

9. A remark on asymptotic behavior. For interesting classes of functions $u$ and operators $P$ one can show a priori that $\lim u(x, t)=0$ as $|x| \rightarrow \infty$ in $\Omega$, uniformly in $t$ for $0 \leqslant t \leqslant T$. The estimation problem then reduces to a similar problem in a bounded region.

In discussing this matter, the first requirement we impose is a condition of integrability:

Definition 3. The function $u \in Z^{m}$ belongs to the class $I$ if $\int_{\Omega} \phi(t,|u(x, t)|) d x<$ $\infty$ for a dense set of values $t>0$, where $\phi: R_{+} \times R_{+} \rightarrow R$ is such that $\phi(t, r \uparrow)$ is strictly increasing and $\phi(t, 0)=0$.

This holds, for example, if in a dense set $\{t\},|u| \in L^{2}(\Omega)$ or $\log (1+|u|) \in L(\Omega)$ or $\exp \left(-1 / t|u|^{2}\right) \in L(\Omega)$. As seen by the third example above, the hypothesis $u \in I$ is much weaker than the assumptions of integrability commonly used, and is not a severe restriction.

More serious is a requirement that the highest derivative shall satisfy a condition of boundedness or uniform continuity, as in the next definition. By a modulus of continuity is meant a function $\phi: R \rightarrow R$ such that $\phi(0+)=0$ :

Definition 4. The function $u \in C^{d} \cap Z^{m}$ belongs to the class $H^{d}$ if one of the following conditions holds for each fixed $T$ :

(i) $D^{\alpha} u$ is bounded in $G(T)$ for $|\alpha|=d$, or

(ii) there exists a modulus of continuity $\phi$ such that

$$
\left|D^{\alpha} u(x, t)-D^{\alpha} u(y, t)\right| \leqslant \phi(|x-y|)
$$

whenever $|\alpha|=d, 0<t \leqslant T$, and $\lambda x+(1-\lambda) y \in \Omega$ for $0<\lambda<1$.

Although the hypothesis $u \in H^{d}$ is rather restrictive, we remark that (i) is appropriate for the theory of strongly coupled systems as developed in [3]-[6], and that (ii) is not out of line with the Hölder condition required in certain existence theories.

Roughly speaking, our third requirement is that the function $f$ in the definition

$$
P u=u_{t}-f\left(x, t, u, u_{x}, u_{x x}, D u, u(\cdot)\right)
$$

shall be bounded when all of its arguments, except $x$, are themselves bounded; e.g., when $0<t \leqslant T, u \in C^{d} \cap Z^{m}$, and $\sup |D u|<\infty$ in $G(T), d \geqslant 2$. If $|P u|$ is 
bounded in $G(T)$, with $f$ and $u$ as described, we deduce that $u_{t}$ is also bounded in $G(T)$. Here $f=\left(f_{1}, f_{2}, \ldots, f_{m}\right)$ and the earlier requirement that only the $k$ th component of $u_{x}$ and $u_{x x}$ shall occur in $f_{k}$ is irrelevant. Nor is any monotonicity condition needed.

Although the following theorem makes no mention of $P$ or $f$, the situation we have in mind is $|P u| \leqslant$ const as discussed above.

Theorem 4. Let $\Omega \in K(\theta, h)$ and suppose $u \in C^{d} \cap Z^{m} \cap H^{d} \cap I$ with $d>1$. Then $|D u|$ is bounded in $G(T)$ for each $T$. If, in addition, $u_{t}$ is bounded in $G(T)$, then for $x \in \Omega$

$$
\lim _{|x| \rightarrow \infty} u(x, t)=0 \quad \text { uniformly in } t, 0<t<T .
$$

Proof. Depending upon which condition (i) or (ii) for the class $H^{d}$ holds, it follows from [11, Theorem 2 or 3], that $\sup |D u|<\infty$ in $G(T)$. Furthermore, it follows from [11, Theorem 4] that $\lim u(x, t)=0$ as $|x| \rightarrow \infty$ in $\Omega$, for the dense set associated with the class $I$. If $\left|u_{t}\right| \leqslant M$ for $0<t \leqslant T$ then

$$
\left|u(x, t)-u\left(x, t_{0}\right)\right| \leqslant M\left|t-t_{0}\right|, \quad 0 \leqslant t, t_{0}<T,
$$

where inclusion of the lower limit 0 is justified by continuity. Given $\boldsymbol{\eta}>0$, cover the interval $[0, T]$ by finitely many intervals $I_{j}$ whose lengths satisfy $0<\left|I_{j}\right|<\eta$. Pick a point $t_{j} \in I_{j}$ and a value $r_{j}$ such that $\left|u\left(x, t_{j}\right)\right|<\eta$ for $x \in \Omega,|x|>r_{j}$. Then $|u(x, t)|<\eta(1+M)$ for $(x, t) \in G(T),|x|>\max r_{j}$. This shows that $\lim u(x, t)=$ 0 uniformly in $t$ and completes the proof.

We shall illustrate Theorem 4 with a class of boundary operators which are difficult to handle, in unbounded regions, by the iterative method used in [12]. The following definition formalizes the ideas discussed informally above:

Definition 5. The function $f: G \times R \times R^{n} \times S^{n} \times\left(C^{d} \cap Z^{m}\right) \rightarrow R^{m}$ belongs to the class $B^{d}$ if the expression

$$
E(x, t)=f\left(x, t, u, u_{x}, u_{x x}, u(\cdot)\right)
$$

is bounded in $G(T)$ whenever $u \in C^{d} \cap Z^{m}$ and $\sup |D u|<\infty$ in $G(T)$.

For example, $f$ could be a polynomial, with bounded coefficients, in the elements of $D u$ or in expressions $F_{i} u$, where each $F_{i}$ is a bounded functional over $C^{d} \cap Z^{m}$. The class $B^{d}$ is closed under formation of sums and products.

Let us consider the interior and boundary operators

$$
\begin{aligned}
& P_{k} u=u_{t}^{k}-f_{k}\left(x, t, u^{k}, u_{x}^{k}, u_{x x}^{k} \uparrow, u(\cdot)\right), \\
& R_{k} u=u^{k}-g_{k}\left(x, t, u^{k}, u_{v}^{k} \uparrow, u(\cdot)\right) \quad(t>0), \\
& R_{k} u=u^{k} \quad(t<0),
\end{aligned}
$$

for $(x, t) \in G$ and $(x, t) \in \Gamma$, respectively. At points of Type $\mathrm{I}$, that is, at boundary points where $u_{\nu}$ is not involved in the boundary condition, the argument $u_{\nu}^{k}$ is dropped from $g_{k}$ and the monotonicity condition is considered to be vacuously fulfilled. Aside from this, $g_{k}$ is a function $\Gamma \times R^{2} \times Z^{m} \rightarrow R$ which vanishes identically when $t \leqslant 0$. 
As continuity conditions we assume

$$
\begin{aligned}
(\operatorname{sgn} z) f_{k}(x, t, z, 0,0, u(\cdot)) & \leqslant \omega\left(t,|z|,|u|_{t},|u|_{\mu, t}\right), \\
(\operatorname{sgn} z) g_{k}(x, t, z, 0, u(\cdot)) & \leqslant \omega_{0}\left(t,|z|,|u|_{t},|u|_{\mu, t}\right),
\end{aligned}
$$

where

$$
\omega(t, \alpha, \beta, \gamma \uparrow) \text { and } \omega_{0}(t, \alpha, \beta, \gamma \uparrow) \text { are monotone. }
$$

Inequalities (14b) are required in $G$ and $\Gamma$, respectively, for $z=u^{k}(x, t), t>0$, and $u$ as in Theorem 4 .

Corollary 5. Let $\Omega \in K(\theta, h), \Omega_{0}=\bar{\Omega}$, and let (14a)-(14c) hold. Suppose further:

(i) For some $d \geqslant 1$ we have $f \in B^{d}, u \in C^{d} \cap Z^{m} \cap H^{d} \cap I$.

(ii) The function $\rho$ satisfies $\rho(0)>\varepsilon(0)$ and $\rho_{t}>\omega\left(t, \rho, \rho, \rho_{\mu}\right)+\delta$, $\rho>\omega_{0}\left(t, \rho, \rho, \rho_{\mu}\right)+\varepsilon, t>0$.

Then $|P u| \leqslant \delta$ in $G$ and $|R u| \leqslant \varepsilon$ in $\Gamma \Rightarrow|u| \leqslant \rho$ in $\bar{G}$.

Proof. It follows from Theorem 4 and from the hypothesis $f \in B^{d}$ that, for $x \in \Omega, \lim _{|x| \rightarrow \infty} u(x, t)=0$ uniformly in $t, 0<t<T$. Hence we can choose $r$ so large that

$$
|x| \geqslant r, \quad x \in G(T) \Rightarrow|u(x, t)|<\inf _{0<t<T} \rho(t) .
$$

If the conclusion $|u(x, t)| \leqslant \rho(t)$ fails there is a Nagumo point $(x, t)$ with $t>0$ and $|x|<r$ at which, without loss of generality, $u^{k}(x, t)=\rho,|u|_{t}=\rho,|u|_{\mu, t}<\rho_{\mu, t}$. The proof of Theorem 1 shows $(x, t)$ cannot be in $G$ and hence $(x, t) \in \Gamma$. From this follows $u_{\nu}^{k} \leqslant 0$, if $u_{\nu}^{k}$ exists, in addition to the relations above. Thus we get a contradiction, $R_{k} u \geqslant \rho-g_{k}(x, t, \rho, 0, u(\cdot))>\rho-\omega_{0}\left(t, \rho, \rho, \rho_{\mu}\right)>\varepsilon$. If $u_{\nu}^{k}$ does not exist, the point $(x, t)$ must be of Type I and the proof simplifies.

\section{REFERENCES}

1. A. Kolmogoroff, On inequalities between upper bounds of consecutive derivatives of an arbitrary function defined on an infinite interval, Učen. Zap. Moskov. Gos. Univ. Mat. 30 (1939), 3-16. (Russian)

2. Karl Nickel, The Crocco-transformation for the three-dimensional Prandtl boundary layer equations, MRC Report 1594, University of Wisconsin, 1975.

3. __ Error bounds and uniqueness for the solutions of nonlinear strongly coupled parabolic systems of differential equations, MRC Report 1596, University of Wisconsin, 1975.

4. __ Fehlerschranken und Eindeutigkeitsaussagan für die Lösungen nichtlinearer, stark gekoppelter parabolischer Differentialgleichungen. Math. Z. 152 (1976), 33-45.

5. __ New results on strongly coupled systems of parabolic differential equations. Proc. Fourth Dundee Conf. on Ordinary and Partial Differential Equations (March 30-April 2, 1976), Lecture Notes in Math., vol. 564, Springer-Verlag, Berlin and New York, 1976.

6. The lemma of Max Müller-Nagumo-Westphal for strongly coupled systems of parabolic functional differential equations, MRC Report 1800, 1977. (German transl. available from the University of Freiburg.)

7. R. M. Redheffer, Die Collatzsche Monotonie bei Anfangswertproblemen, Arch. Rational Mech. Anal. 14 (1963), 196-212.

8. R. M. Redheffer and W. Walter, Existence theorems for strongly coupled systems of partial differential equations over Bernstein classes, Bull. Amer. Math. Soc. 82 (1976), 899-902.

9. R. M. Redheffer, Uniqueness, stability and error estimation for parabolic functional-differential equations, Ber. 9, Univ. Karlsruhe (1976). Accepted by the Soviet Academy of Sciences in October, 1976 for the Jubilee Volume dedicated to the 70th anniversary of Academician I. N. Vekua. 
10. Das Maximumprinzip in unbeschränkten Gebieten für parabolische Ungleichungen mit Funktionalen, Math. Ann. 226 (1977), 155-170.

11. ___ Inequalities involving derivatives, Pacific J. Math. 85 (1979), 165-178.

12. Comparison theorems for parabolic functional inequalities, Pacific J. Math. 85 (1979), 447-470.

13. Nonuniqueness of the null solution for strongly coupled systems of parabolic differential equations, Math. Z. 171 (1980), 83-90.

14. J. Szarski, Differential inequalities, Monogr. Mat., Tom 43, Warsaw, 1965.

15. W. Walter, Differential and integral inequalities, Ergebnisse der Mathematik und ihrer Grenzgebiete, Bd. 55, Springer-Verlag, Berlin and New York, 1970. (Further references to the early history can be found here.)

Department of Mathematics, Universtty of California, Los Angeles, California 90024

Mathematical Institute, University of Karlsrufe, KarLsRUhe, Federal Repubuc of Germany 